\title{
GESTIÓN Y DESARROLLO DE PLANES DE ACCIÓN TUTORIAL EN LA UNIVERSIDAD. ESTUDIOS DE CASOS
}

\section{MANAGEMENT AND DEVELOPMENT OF ACTION TUTORIAL PLANS AT THE UNIVERSITY. CASE STUDIES}

\author{
Luis Venegas - Ramos ${ }^{1}$ \\ Joaquín Gairín Sallán \\ Universidad Autónoma de Barcelona, España.
}

\section{RESUMEN}

El objetivo del estudio es analizar experiencias consolidadas de Planes de Acción Tutorial (PAT) con el fin generar referencias para la construcción y mejora de modelos de intervención sobre tutoría universitaria.

La investigación parte del análisis de 16 planes tutoriales de 14 universidades y se profundiza en un estudio de casos múltiples de seis universidades españolas, específicamente de las Comunidades Autónomas de Cataluña, Valencia, Aragón y País Vasco, donde se desarrollaron entrevistas convergentes en profundidad. Los datos fueron procesados mediante la herramienta informática Max QDA, que permitió generar 418 unidades de significado, divididas en 8 categorías de análisis que se asocian al desarrollo y gestión de los PAT.

Los resultados indican que la cultura universitaria, el rol de las autoridades y los modelos de orientación educativa y de estructura organizativa que adoptan las instituciones analizadas para dar soporte al proceso de acción tutorial, ayudan o interfieren en el desarrollo e impacto de los PAT, lo que evidencia la necesidad de repensar la forma como se gestiona la tutoría universitaria en la enseñanza superior. También evidencia la existencia de sistemas de funcionamiento piramidales y colaborativos, y una combinación de los modelos clínico, de programas y de servicios que aparecen con más fuerza en función del tipo de articulación que se considera en el PAT.

\footnotetext{
1 Correspondencia: Luis Venegas-Ramos: Departament de Pedagogia Aplicada, Plaça del Coneixment (Edifici G6-247) Campus Bellaterra, C.P 08193, Bellaterra (Cerdanyola del Vallès). Barcelona, España. Luis.Venegas@uab.cat
} 
Palabras Clave: plan de acción tutorial, tutoría, orientación universitaria, gestión.

\section{ABSTRACT}

The objective of this study was to analyze successful Tutorial Action Plans (TAP) from different institutions, and to generate a reference framework for the creation of a management model for university tutoring.

The research was based on case studies from six universities in northeastern Spain, specifically from the Autonomous Communities of Catalonia, Valencia, Aragon and Basque Country, where in-depth interviews were conducted. A qualitative approach was used and the obtained data was processed using the text analysis software Max QDA. This analysis yielded 418 units of meaning, divided into 8 different categories of analyses that were associated with development and management of TAPs.

The results from the generated reference framework showed that, the development and impact of TAPs in the analyzed institutions is either positively or negatively affected by: their culture, the role of their authorities, the educational counseling models and the organizational structure that they adopt to support the process of tutorial action. These findings suggest the need of a re-evaluation of the management methods used on university tutoring. The evidence also indicates the existence of pyramidal and collaborative functioning systems, and a combination of clinical models, programs and services that appear more strongly depending on the type of articulation considered in the TAP.

Key Words: tutorial action plan, tutoring, university counseling, management.

\section{Introducción}

La acción tutorial se considera una de las estrategias de mejora más importantes en la enseñanza superior, vinculada a la calidad del proceso formativo del estudiante (Miraflores, 2009. Cit. en Blanchard, 2010). Sin embargo, "el éxito del plan para llevarla a cabo, no sólo reside en su contenido y su filosofía, sino que también, en la manera de organizarse, en la coherencia con las políticas institucionales, y en la estimación de condiciones adecuadas que cualquier proceso de innovación y cambio debe considerar" (Gairín, Guillamón, Quinquer, \& Feixas, 2005, p. 35)

Los Planes de Acción Tutorial (PAT, en adelante) han de concebirse y presentarse, en este sentido, secuencialmente estructurados, para ser eficaces y eficientes, dentro de unas coordenadas espacio-temporales bien definidas y con proyección en la trayectoria universitaria de sus destinatarios, desde el Bachillerato a la Universidad, finalización de los estudios e inserción laboral (Cano, 2008).

Asimismo, Álvarez (2014) señala la necesidad de fomentar el diseño y formalización de los PAT basados en un contexto concreto y a partir de un verdadero análisis de necesidades; también, que se deben instrumentar acciones para una adecuada evaluación del PAT tomar decisiones que permitan mejorar dicha planificación y poder desempeñar sus objetivos, reorientando el modelo tutorial existente.

Esta situación invita a reflexionar y analizar como las instituciones universitarias gestionan y despliegan sus Planes de Acción Tutorial como concreción de la orientación universitaria que desarrollan. Máxime cuando la existencia o no de los PAT y su funcionamiento son uno de los 
indicadores importantes que contempla a acreditación de titulaciones que realiza la Agencia Nacional de Evaluación de la Calidad y Acreditación (ANECA).

\section{Antecedentes y fundamentación}

Los cambios producidos por la convergencia europea han cambiado el papel de la tutoría universitaria como innovación en la formación integral de los estudiantes de enseñanza superior (Martínez, Martínez y Pérez, 2014). Actualmente, se la implica en el conocimiento del alumnado, la creación de entornos de aprendizaje, el conocimiento y el acceso a los recursos de la institución, la creación de recursos humanos y materiales orientados a los nuevos roles y el establecimiento de programas de formación de tutores, estando también alerta a los problemas personales que puedan inhibir el aprendizaje de los estudiantes y ayudando a reconsiderar las políticas internas de los centros de estudio (Sanz, 2005).

Figuera y Álvarez (2014) señalan además, que producto de estos cambios, los sistemas de evaluación y acreditación incluyen como indicador clave los procesos de orientación y tutoría, como uno de los instrumentos más poderosos para optimizar el proceso de transición a la universidad y la promoción del estudiante universitario.

No obstante, "ha habido una tendencia a asimilar la tutoría casi exclusivamente con la orientación puramente académica, en relación a la materia que imparte el profesor y específicamente con la resolución de dudas antes de los exámenes y después para la revisión de los mismos" (Martínez et al., 2014, p. 132). Así, se ha impulsado la reflexión continua sobre el proceso de implantación y desarrollo de la acción tutorial en los centros universitarios, que, en palabras de De la Rubia, Tur, Paz, Álvarez y Guillem (2013, p. 2) debe entenderse como "un elemento dinamizador para que todos los subsistemas de la organización educativa de la Universidad apoyen al estudiante para conseguir que este sea el agente activo de su aprendizaje".

Durante estos últimos quince años se ha discutido así sobre la forma de cómo ha de desplegarse el sistema de acción tutorial, tomando en cuenta las modalidades a desarrollar, los recursos para llevarla a cabo, la manera como han de organizarse los actores involucrados y la manera de planear y evaluar dicho sistema (Gairín, Feixas, Franch, Guillamón y Quinquer, 2003; Rodríguez, 2004; Gairín et al., 2005; Cano, 2008; Rumbo y Gómez, 2011; Álvarez, 2014; Álvarez y Álvarez, 2015), con el consenso de que las estrategias para la implementación se concretarán a través de un Plan de Acción Tutorial².

El Ministerio de Educación y Cultura de España, ya hablaba en los años noventa del PAT como "el instrumento por excelencia para la planificación de la tutoría, el cual constituye un marco en el que se especifican los criterios de la organización y las líneas prioritarias de funcionamiento de la tutoría en el centro educativo" (MEC, 1995. Cit. en Blasco y Pérez, 2012). En la universidad a partir del acuerdo de Bolonia se observan aportaciones que incluyen aspectos adicionales para su mayor comprensión y ejecución, que se relacionan con los siguientes elementos generales (Rodríguez, 2004; Gairín et al., 2005; Sanz, 2009; Álvarez, 2014) que habría que considerar:

- Diseño: establecimiento de necesidades del alumnado y de la institución, objetivos a perseguir, dimensiones o ámbitos a desarrollar y recursos materiales y humanos a disponer.

- Implementación: contenidos a tratar, estrategias didácticas, tipología de tutoría a desarrollar y momentos clave del proceso.

\footnotetext{
${ }^{2}$ También denominado plan de tutoría universitaria, y en otros casos, plan de orientación y acción tutorial.
} 
- Evaluación: momentos, técnicas, instrumentos y alcances.

- Organización: modelo organizativo a desplegar, coordinación del plan, selección de los tutores según las modalidades, formación y otros soportes, seguimiento del plan, servicios educativos y de orientación implicados en dicho proceso.

Esta estructura por sí sola no asegura que la acción tutorial a desarrollar en la universidad tenga el logro que se espera, pues como señala Gairín et al., (2005), el éxito del PAT depende de varios factores tales como: i) la coherencia del plan tutorial con las políticas institucionales, ii) la consideración del plan tutorial como un elemento de innovación, y iii) la consideración de los resultados de la acción tutorial para fomentar el debate, la reflexión y la mejora organizacional.

$Y$ es que los elementos generales del PAT a menudo indican que el sistema se inspira en el proceso clásico de dirección, planificación, coordinación, motivación y control (Alcolea y Pérez, 2012), que puede ser muy normativo y funcional pero que no refleja completamente las complejidades organizativas que se presentan, se centra muy poco en las relaciones informales entre responsables y ejecutores y no considera el cómo desarrollar y mantener una fuerza de trabajo motivada (Riquelme, 2014).

Álvarez y Álvarez $(2012 ; 2015)$ han recogido, al respecto, evidencias sobre las debilidades del modelo actual de tutoría (o modelo tradicional) remarcando la falta y/o la escasez de: una planificación y desarrollo efectivo del PAT en las titulaciones, una estructura metodológica y de gestión adecuada para el desarrollo de la función tutorial, el reconocimiento de la labor del coordinador de tutoría por los responsables académicos de la carrera y la relevancia que se debe dar a la tutoría de asignatura. Así mismo señalan que existe poca tradición en el uso de las TIC para el desarrollo de los PAT, en la gestión y el seguimiento del alumnado y una falta de coordinación entre los agentes y los servicios universitarios implicados. Posteriormente, Álvarez (2017) incorpora nuevas consideraciones:

- Marco Institucional: Las políticas institucionales favorecen los procesos de mejora y cambio, pero se confirma la falta de planificación y diseño de los PAT en algunos centros y falta de tradición en procesos de orientación y seguimiento del estudiante.

- Planificación del PAT: Falta de organización para que la tutoría sea operativa, que se traduce en falta de coordinación, de tiempo y espacios para la tutoría, a pesar de que la concreción de los PAT surja desde la realidad concreta de donde se aplica.

- Acompañamiento de la Tutoría: Poca implicación del profesorado y falta de coordinación entre los tutores.

- Difusión a los Estudiantes: Desconocimiento del PAT por parte de los estudiantes y expectativas de los mismos que no se cumplen en los PAT.

Aunque las universidades españolas han ido desarrollando de manera paulatina y creciente sus propios modelos de organización para apoyar sus PAT y rediseñan y adecúan este instrumento a las realidades de cada facultad y de cada titulación, cabe preguntarse ¿cuál es el paradigma de orientación y tutoría que direccionan los PAT?, ¿qué implicaciones tiene para los distintos agentes involucrados?, ¿cuál es el modelo organizativo que adopta la institución para apoyar sus PAT?, y ¿en base a qué procesos ha ido evolucionando el sistema de acción tutorial al interior de la institución?

Las cuestiones son importantes si pensamos que la tutoría "reaparece como un elemento inherente y consustancial a la función docente, como un factor de concreción práctica de la actividad orientadora de carácter integral; y en definitiva, como un elemento de calidad y excelencia de la Educación Superior" (Cano, 2008, p. 188), llegando incluso a establecerse como indicador de acreditación institucional. Como señalan Rumbo y Gómez (2011), el PAT ha de concebirse como: 
... una propuesta de actuación para integrar la cultura de la tutoría en la universidad española, intentando afrontar y solventar los principales obstáculos y desarrollando medidas para la mejora de la calidad de la misma, haciendo especial hincapié en la participación de los agentes implicados en dicho proceso, docentes y estudiantado, con la finalidad de construir una universidad más democrática en el marco europeo de educación superior (p.24).

La acción tutorial en este contexto, ha pasado de ser la visualización del compromiso con los cambios políticos y curriculares y una respuesta estratégica de las instituciones para la atención de colectivos vulnerables, a ser una función universitaria de carácter primordial para el desarrollo del proceso de enseñanza - aprendizaje (en los distintos momentos de la vida universitaria) y un elemento que aporta calidad al sistema, remarcando que la manera de gestionarla ha de implicar cambios clave a nivel institucional.

Esta perspectiva se alinea, por otra parte, con los procesos tutoriales desarrollados en otros sistemas universitarios como expresan las siguientes experiencias:

- En Italia, la acción tutorial se suscribe en el marco de orientación permanente propuesta por el Estado, que ha institucionalizado recursos y soportes técnicos en el Ministero dell'Istruzione, dell'Universita e della Ricerca para la promoción de un sistema integrado y eficaz de orientación antes, durante y después de la vida universitaria, con mayor atención en la transición al mundo laboral mediante Servicios de Tutoría dependientes de cada universidad (Da Re, 2013; Traverso, 2015).

- En Francia, se da una articulación entre los servicios universitarios de información y orientación dependientes de cada universidad, con los servicios sociales de la comunidad o región en la que estén insertas, que ofrecen servicios relacionados con el bienestar personal de los estudiantes (Vieira, 2007).

- La tutoría universitaria, países como Reino Unido y Estados Unidos, está centrada primordialmente en la atención biopsicosocial del estudiante y la orientación vocacional, de la creación de Departamentos de Servicios de Estudiantes y/o Divisiones de Asuntos de Estudiantes (Vieira, 2007). Así mismo en Reino Unido, cada escuela académica o facultad, posee un responsable docente de la coordinación general y apoyo al sistema tutorial o "senior tutor", quien oficia como puente entre los departamentos, las escuelas académicas y los servicios de estudiantes (Luck, 2010).

Finalmente, cabe destacar como el contexto cultural y socioeconómico de cada país, la variedad de instituciones de educación superior y las condiciones de acceso a las mismas, la tradición del sistema educativo de ofrecer programas y servicios de apoyo, el grado de autonomía y financiación de las instituciones, y principalmente, las características de los estudiantes que ingresan a la universidades, se transforman en factores claves a la hora de planificar y organizar el sistema de apoyo y orientación al estudiante (Vieira, 2007), complejizando así el terreno para el diseño de las actuaciones tutoriales.

\section{Método}

La investigación adoptó una orientación cualitativa mediante estudio de casos múltiples, que resulta apropiada al tema en estudio (Creswell, 2013) y sirve para analizar aquellos problemas 0 situaciones donde se presentan diferentes factores que determinan su evolución y que están estrechamente vinculados al contexto donde se desarrollan (C. Álvarez y San Fabián, 2012). Buscando obtener las perspectivas y puntos de vista de los participantes de determinados fenómenos (Hernández, Fernández y Baptista, 2006, p. 8), se utilizó la entrevista (individual y en 
grupo) como técnica de recolección de datos, focalizadas en cómo se gestiona la acción tutorial al interior de instituciones de educación superior.

\section{Procedimiento para la selección de los Casos}

Inicialmente, se recopiló información pública de las universidades españolas sobre sus planes de acción tutorial. La tutoría como derecho de los estudiantes (reconocido en R.D 179/2010, de 30 de diciembre, por el que se aprueba el Estatuto del Estudiante Universitario), se refleja en las páginas web de las distintas instituciones -como también en publicaciones en revistas especializadas de educación superior- donde se pueden identificar los elementos básicos de sus PAT según estén diseñados (a nivel universidad, a nivel de facultad, o a nivel de titulación).

La selección de los PAT consideró las universidades que participaron en el Simposio "Sistemas de Acogida y Tutorización en Estudios Universitarios -SATEU 2013". Así mismo, se revisaron los de las universidades ubicadas en Ranking ISSUE (Indicadores Sintéticos del Sistema Universitario Español) de 2014, específicamente, en el Top 10 del ISSUE -V v/s Shanghai.

Los 16 planes tutoriales de 14 universidades analizados permitieron conocer la tipología de acciones tutoriales adoptadas, la planificación y desarrollo de los planes tutoriales, la vinculación con el modelo educativo institucional y sus implicaciones en los distintos niveles de funcionamiento universitario. Se utilizó, al respecto, una pauta de cotejo con 21 indicadores de carácter dicotómico segmentados de acuerdo, los siguientes criterios:

- Contenido de la información obtenida o centralidad del artículo o recurso web revisado (descripción del PAT, evaluación del PAT, estrategias del PAT, gestión del PAT y Diagnóstico para la implementación del PAT.

- Modalidad tutorial implementada (docente - par u otras).

- Tipos de tutoría (académica, de la carrera, personal, profesional, integral).

- Organización institucional para el PAT (piramidal, inter estamentos).

- Estructura general del PAT (diseño, procesos, desarrollo, evaluación).

- Evaluación del proceso (momentos, procedimientos, instrumentos).

El estudio documental realizado permitió seleccionar un número discreto de universidades para establecer los estudios de caso, que proporcionaban información sobre, como mínimo, como mínimo diez indicadores propuestos en la pauta de cotejo, sin que fuera predominante la titularidad institucional (pública/privada). Fueron seis las universidades seleccionadas (pertenecientes a las Comunidades Autónomas de Cataluña, Valencia, Aragón y País Vasco) ${ }^{3}$, que reunían los atributos siguientes:

- Descripción detallada en sus PAT respecto de los roles y funciones de los responsables del sistema (gobierno universitario, responsables de facultades, coordinadores de tutorías, servicios universitarios).

- Estrategias a considerar en el diseño de los PAT (difusión, contenidos, formación de tutores, integración al currículum).

\footnotetext{
${ }^{3}$ Las universidades privadas U. San Jorge y U. de Deusto, junto a las universidades públicas U. Pompeu Fabra, U. de Valencia, U. de Barcelona y U. Autónoma de Barcelona, fueron aquellas que se ajustaron al criterio de selección para iniciar los estudios de caso y aceptaron bajo consentimiento informado dicha participación.
} 
- Formas de colaboración con otros servicios universitarios y recursos disponibles para concretar la acción tutorial.

- Detallada descripción de las acciones a concretar en los distintos momentos del curso académico (inicio del curso, avance, exámenes, etc) y del proceso universitario (acceso a la educación superior, permanencia en la universidad, egreso al mundo laboral).

\section{Selección de los Informantes}

Los informantes seleccionados en cada caso, se rigió por el método de muestra de casos tipo, utilizada generalmente en estudios con perspectiva fenomenológica, donde el objetivo es la riqueza, profundidad y calidad de la información que permiten adentrarse en el análisis de los patrones y/o formas de actuación respecto de un fenómeno en cuestión (Hernández, Fernández, \& Baptista, 2010). Se buscó la pertinencia y experticia en la conducción y desarrollo de la acción tutorial en sus respectivas instituciones y su vínculo con el diseño e implementación de los PAT, lo que responde a uno de los factores propuestos por Hernández et al., (2010) para la determinación del número de casos o tamaño de la muestra a utilizar: el entendimiento del fenómeno en cuestión.

A raíz de ello se contó con la participación de nueve agentes relevantes para el total de los casos (ver tabla 1), considerando los criterios antes expuestos. El contacto partió de solicitud formal institucional (enviada por correo electrónico) y el establecimiento posterior de citas presenciales. Bajo la técnica de entrevistas convergentes, se buscó contrastar datos entre los distintos informantes y completar la información máxima sobre los PAT de sus respectivas instituciones.

TABLA 1. Fuentes de información de las unidades de análisis

\begin{tabular}{ll}
\hline \multicolumn{1}{c}{ Tipo de fuente } & \multicolumn{1}{c}{ Participantes } \\
\hline Autoridades a nivel institucional (2) & Vice rectora de Ordenación Académica, Vice decana de \\
& Ordenación Académica. \\
Responsables de los PAT (3) & Profesionales del ámbito de gestión administrativa y de \\
& recursos del área de calidad institucional, Profesionales \\
Ejecutor PAT (1) & para el ámbito de dinamización y orientación al estudiante. \\
Responsables de Formación Docente (3) & $\begin{array}{l}\text { Profesor/Tutor titular } \\
\text { universidad }\end{array}$ \\
\hline
\end{tabular}

Instrumento de Recolección y Análisis de Datos

Las entrevistas convergentes se focalizaron en una serie de elementos de análisis como referente, pero su desarrollo fue abierto, con la finalidad de conocer tanto la realidad como los porqués de la misma y las consecuencias (efectos, problemáticas y alternativas) que se derivan de las opciones realizadas, estructurándose en base a una matriz de 18 preguntas abiertas (ver anexo 1), de acuerdo a los siguientes tópicos: 
TABLA 2. Estructura de Entrevistas en profundidad

\begin{tabular}{lllc}
\hline \multicolumn{1}{c}{ Componente } & \multicolumn{1}{c}{ Sub Componente } & \multicolumn{1}{c}{ Elemento de Análisis } & Cantidad de preguntas \\
\hline $\begin{array}{l}\text { Elementales relacionados } \\
\text { con la Acción Tutorial }\end{array}$ & Concepciones & Orientación Universitaria & 1 \\
& $\begin{array}{l}\text { Bases } \\
\text { Implicancias del Servicio } \\
\text { (para...) }\end{array}$ & $\begin{array}{l}\text { Tutoría Universitaria } \\
\text { El Estudiante }\end{array}$ & 1 \\
& & El Profesorado & \\
Elementales sobre el Plan & Organización para la & Estructura organizativa del PAT & 1 \\
de Acción Tutorial (PAT) & Acción Tutorial & Funciones Actores Involucrados & 1 \\
& Tipo de PAT & Participación de las Autoridades & 1 \\
& & Tipología & 1 \\
Sobre la Gestión de la & Historial del Proceso & Planes Específicos & 1 \\
Acción Tutorial & Estrategias de Desarrollo & 1 \\
& Gestión Base & Planes Complementarios & 1 \\
& \multirow{2}{*}{ Implantación } & 1 \\
& Desarrollo y Seguimiento & 2 \\
\hline
\end{tabular}

Fuente: Elaboración Propia

En total se entrevistaron en profundidad a 4 informantes de manera individual y a 5 de forma grupal (dos entrevistas de 2 y 3 sujetos cada una, según las posibilidades de la institución participante); lo que permitió obtener una visión más cercana del fenómeno en encuentros de 1 a $1 \frac{1}{2}$ horas por universidad.

Finalmente se obtuvieron sobre 06 horas 30 minutos de grabación, que fueron transcritas íntegramente y analizadas a partir del programa MaxQDA con la finalidad de obtener unidades de significado y generar categorías de análisis.

El análisis de la información partió de una segmentación de los datos transcritos, tomando en cuenta aquellas frases o fragmentos (unidades de significado) que reflejaran las "ideas fuerza" bajo un sistema de códigos o categorías emergentes. El proceso de construcción de categorías fue de carácter inductivo, abierto y generativo. A medida que se realizaba una nueva lectura de las transcripciones, cada unidad de significado se correspondía a un código o generaba otra categoría emergente, hasta alcanzar una saturación de categorías. De esta manera se establecieron 418 unidades de significado agrupadas en 8 categorías emergentes distribuidas según indica la tabla 3 :

TABLA 3. Distribución y peso de las unidades de significado

\begin{tabular}{llccc}
\hline $\begin{array}{c}\text { Código } \\
\text { Abreviado }\end{array}$ & \multicolumn{1}{c}{ Categoría Emergente } & $\begin{array}{c}\text { Segmentos } \\
\text { Codificados }\end{array}$ & $\begin{array}{c}\text { \% } \\
\text { Codificaciones }\end{array}$ & Documentos \\
\hline 1_PrAT & Paradigma de Acción Tutorial. & 27 & 6,46 & 6 \\
2_BPpAT & Buenas Prácticas para la Acción Tutorial. & 143 & 34,21 & 6 \\
3_DIAT & Dificultades de implementación de Acción Tutorial & 62 & 14,83 & 6 \\
4_EAT & Evaluación y Acción Tutorial. & 40 & 9,57 & 6 \\
5_FyST & Formación y Soporte a la Acción Tutorial. & 28 & 6,70 & 6 \\
6_PAU & Papel de la Autoridad Universitaria. & 24 & 5,74 & 6 \\
7_IBP & Ideal de Buenas Prácticas. & 34 & 8,13 & 6 \\
8_CUAT & Cultura Universitaria y Acción Tutorial. & 60 & 14,35 & 6 \\
\hline \multicolumn{2}{c}{ Fuente: Elaboración Propia. } \\
\hline
\end{tabular}




\section{Resultados}

En una primera aproximación (ver tabla 3), se puede identificar que las mayores frecuencias (f) se encuentran asociadas a las categorías emergentes "Buenas prácticas para la Acción Tutorial" (f: 143), "Dificultades de implementación de Acción Tutorial" (f: 62) y "Cultura Universitaria y Acción Tutorial" (f:60), a diferencia del resto de las categorías que se visualizan con frecuencias más moderadas. Otros resultados según categorías, asignando los códigos correlativos UA-01 hasta UA-06, a las universidades participantes se presentan a continuación.

\section{Paradigma de Acción Tutorial}

Esta categoría aparece como un elemento vertebrador de la planificación y gestión del PAT. Los participantes del estudio coinciden en que la orientación y la tutoría son acciones que van juntas y que dependen una de la otra, pero, a su vez, visualizan la acción tutorial como una herramienta o un ejercicio mediante el cual se concreta la orientación universitaria.

"la orientación y la tutoría son dos elementos que vemos íntimamente relacionados"

[UA-05; Posición: 3-3; 22-02-2016 1:45]

"para mí la tutoría es una herramienta complementaria siempre dentro de un marco mucho más general que es el marco de la orientación, que se puede dar por distintos agentes en distintos momentos a lo largo de toda la carrera"

[UA-06; Posición: 7-7; 22-02-2016 1:45]

Considerando los modelos de orientación educativa propuestos por Duarte, Vasconcelos, Asencio, Martínez \& Laguna (2012), se puede señalar que las universidades estudiadas se mueven en tres modalidades:

- Modelo clínico o counseling (UA- 01, 02, 03, 04, 05, 06), que se centra en atender las necesidades del individuo en los aspectos personales, educativos y socio-profesionales, a través de consultas a sus profesores.

- Modelo de Programas (UA-01; UA-03; UA-05), que pretende actuar en el contexto educativo mediante actividades curriculares previamente planificadas y coordinadas con el currículum en el centro educativo y/o con el programa oficial de estudios.

- Modelo de Servicios (UA-03; UA-06), que desarrolla sus acciones mediante la intervención directa de un equipo o servicio especializado sobre un grupo reducido de sujetos, para satisfacer necesidades de carácter personal y educativo, a partir de estrategias clínicas.

Es preciso señalar que las universidades consultadas no se posicionan exclusivamente en un modelo de orientación, sino que van apareciendo estas formas de actuación a medida en que se articulan en niveles específicos de intervención. Así, el counseling aparece cuando la articulación alcanza el nivel de titulación; a nivel de facultad o centro emerge el modelo de programa y se mantiene el anterior; y a nivel de universidad se suma el modelo de servicios. 


\section{Buenas prácticas para la Acción Tutorial}

Las universidades, valoran, sobre todo, aquellas acciones que permiten coordinar Planes de Acción Tutorial bajo una organización de carácter integral a nivel toda la institución, considerando distintos representantes de las facultades, de los servicios complementarios y de formación docente, del gobierno universitario y estudiantes delegados. Consideran, asimismo, la necesidad de concretar procesos de evaluación para la mejora continua de los programas, tal y como nos indica una de las fuentes de información:

"aquello que no se evalúa no se sabe qué utilidad tiene y difícilmente se puede mejorar sólo a base de intuiciones o a creencias"

[UA-06; Posición: 15-15; 22-02-2016 1:45]

Se trata de utilizar herramientas tecnológicas para el seguimiento y generación de informes anuales, encuentros con los estudiantes, con los docentes y con los representantes del gobierno universitario para ir avanzando en materia de cooperación inter estamentaria.

Otros elementos a destacar serían no perder de vista la permanencia del estudiante entendiendo que la tutoría ha de ser un sistema continuo; sistematizar lo que se evalúa; investigar y evaluar durante el proceso; combinar tutoría docente con el sistema mentoring; y generar áreas de gestión específicas para el desarrollo de la acción tutorial al interior de la universidad.

\section{Dificultades de implementación de la Acción Tutorial}

El panorama de los elementos que obstaculizan un buen desarrollo de los PAT sería el siguiente:

- A nivel institucional, aunque la acción tutorial está normada y es un proceso común en las universidades, no posee la misma relevancia respecto de otras funciones universitarias tales como la investigación y la docencia.

"la primera necesidad es cubrir la docencia, o quizás es la segunda, porque la primera necesidad es cubrir la investigación, entonces cuando un profesor hace su investigación y cubre además docencia, no le queda más tiempo para cubrir tutorías. La tutoría es importante pero no la apreciamos aún como una primera necesidad"

[UA-01; Posición: 37-37; 22-02-2016 1:45]

"los profesores no tienen ningún reconocimiento por este trabajo, cada facultad decide, propone, ordena, hay de todo"

[UA-06; Posición: 46-46; 22-02-2016 1:45]

- Hay dificultades relacionadas con la inyección económica que merece la acción tutorial, relacionadas con la remuneración docente y el financiamiento de nuevas acciones y materiales que favorecen la puesta en marcha de los PAT. 
"en los planes estratégicos aparece siempre el plan de acción tutorial priorizado pero no se inyectan recursos para que pueda crecer y avanzar"

[UA-02; Posición: 76-76; 22-02-2016 1:45]

"se hicieron planes de acción tutorial más vinculados al profesorado y más conectados con lo académico, se acaba el dinero, se acaba el programa"

[UA-06; Posición: 69-69; 22-02-2016 1:45]

- La motivación docente para ejercer el rol de tutor en base a cuestiones de reconocimiento económico, el desinterés de los estudiantes hacia el sistema tutorial y la falta de formación ad hoc para los fines de la orientación universitaria, son también dificultades a considerar.

"hay profesores que les cuesta mucho esfuerzo por que no tienen unas habilidades y les cuesta, además, considerando al tipo de carrera a la que pertenezca"

[UA-03; Posición: 13-13; 22-02-2016 1:45]

"la sensación del fracaso que llegan a tener los mentores cuando sus mentorizados no les contestan, no vienen a sus convocatorias, no responden sus correos o no contactan con ellos, es una de las principales dificultades que tenemos"

[UA-06; Posición: 71-71; 22-02-2016 1:45]

- La descoordinación entre centros e intrainstitucionalidad dificulta el que las acciones de orientación universitaria no se cumplan en su totalidad o por lo menos como están planeadas, como ocurre con la falta de procesos de evaluación para la mejora continua.

"hay centros que van haciendo actividades sueltas, pero no van dando una posición global al PAT"

[UA-02; Posición: 14-14; 22-02-2016 1:45]

"aquí, el servicio de atención a los estudiantes continua asesorando a los estudiantes independientemente de los profesores y los tutores continúan haciendo su función sin "hablarse" con el servicio de atención a los estudiantes"

[UA-02; Posición: 41-41; 22-02-2016 1:45]

"en cuanto a organización en algunas facultades, no quedaba claro de quién dependía la coordinación del PAT dentro de la facultad"

[UA-04; Posición: 20-20; 22-02-2016 1:45]

\section{Cultura Universitaria y Acción Tutorial}

Se vincula este aspecto con el interés y la motivación tanto del docente como del estudiante respecto de la participación en el sistema de acción tutorial. Un efecto prácticamente obvio que se constata es que una mayor implicancia del docente en el asesoramiento de un estudiante genera 
mayor rapport a la situación de acompañamiento y una percepción más positiva de la universidad; y viceversa: a mayor implicancia del estudiante con el proceso de acompañamiento y orientación, el docente se ve menos frustrado y con mayor implicación en el desarrollo del estudiante y con el proyecto educativo institucional.

Los aspectos anteriores forman parte de la cultura organizativa, como también las creencias que poseen, los valores y las presunciones básicas compartidas, como proponen Folch e lon (2008, p. 133. Cit. a Rossman et. al., 1988; Schein, 1992); no obstante, para describir este punto, quizás es preferible que los mismos participantes del estudio hablen por sí solos respecto de la significancia de la acción tutorial y su trascendencia:

"la universidad es una experiencia de vida no solo de asuntos académicos, sino también sobre las relaciones que se establecen con los profesores y con los estudiantes"

[UA-04; Posición: 16-16; 23-02-2016 13:43]

"para nosotros, la atención personalizada del estudiante forma parte del proyecto formativo. Nos interesa formar buenas personas y que sean profesionales y por eso estamos sustentados en un planteamiento de identidad basado en humanismo cívico y enfocamos nuestro proyecto formativo desde este plan estudiantil de acción"

[UA-05; Posición: 3-3; 23-02-2016 13:43]

"ha habido un cambio, al menos lo que es la interfaz de la universidad con la sociedad, de manera que eso de la universidad centrada en el estudiante deja de ser una cosa abstractaal menos en la interfaz- y poco concreta. Cuando entras a la página web de la universidad, la primera pestaña con la que te encuentras es con futuros estudiantes o estudiantes de la universidad, reflejando que hemos trabajado mucho y que debemos seguir avanzando a la idea de que la universidad está al servicio de la sociedad"

[UA-06; Posición: 103-103; 23-02-2016 13:43]

"Es una labor que beneficia a la institución en términos de aprendizaje organizacional"

[UA-04; Posición: 11-11; 22-02-2016 1:45]

\section{Evaluación y Acción Tutorial}

La evaluación del despliegue de los PAT es uno de los elementos que se reconoce como "pendiente" por los informantes. En general, no se generan procesos evaluativos muy profundos y los que se efectúan van en la línea de la valoración de la acción tutorial concreta (encuentro estudiante - docente). Esta focalización puede explicar el que los cambios producidos en los PAT no son mayores. Así, se pone de manifiesto que la evaluación de la acción tutorial se realiza: i) de manera indirecta a través de la evaluación de las asignaturas donde los profesores además ejercen de tutor y que, en general, dependen de cada facultad (UA-01; UA-02); ii) de manera directa, a través de cuestionarios de valorización integrados en la evaluación docente (UA-05) o de manera separada (UA-03; UA-06); ó iii) no se evalúa y más bien se consulta de manera informal a los tutores implicados (UA-04).

En el caso de las unidades de análisis UA-03, UA-04 y UA-06, se han incorporado aplicativos intranet para los procesos de evaluación y seguimiento del PAT y de los estudiantes, que ofrecen estadísticas y valoraciones nominativas que permiten generar informes al final de cada curso para la toma de decisiones; sin embargo, su buena realización amerita una dedicación especial para su sostenibilidad y un equipo constante para evitar el desuso del aplicativo. 


\section{Formación y Soporte a la Acción Tutorial}

Los soportes para la acción tutorial se mueven en dos direcciones en las universidades consultadas. Por un lado, existen soportes a cargo de unidades y/o servicios especializados en orientación (UA-03; UA-05), dinamización de estudiantes (UA-06), y/o gestión de la calidad (UA04), y por otro, soportes que dependen de cada facultad (UA-01; UA-02).

El primer tipo de soporte permitiría una conexión fuerte entre las distintas facultades y titulaciones con el nivel central de la universidad y en especial con los profesores tutores, la coordinación del centro y de las titulaciones, ya que serían estas mismas unidades o servicios los encargados de formar al docente en el ámbito de la acción tutorial y de acompañarlos durante el curso en desarrollo.

El segundo tipo de soporte se percibe como más laxo y con poca implicancia del nivel central y estaría a cargo de una comisión de docencia, o en su defecto, del coordinador de titulación. A pesar de ello, permitiría mayor autonomía a las facultades y sus titulaciones para formarse en los ámbitos de interés que atañen a su contexto local. La formación de tutores sería desarrollada a través de unidades encargadas de la calidad institucional o a partir de seminarios internos de cada facultad (UA-01), o bajo la responsabilidad de unidades de desarrollo docente (UA-02).

\section{Papel de la Autoridad Universitaria}

El papel que ejercen las autoridades de la universidad respecto de la implementación y el desarrollo de los PAT depende, principalmente, de la manera cómo el plan esté diseñado y cómo exprese las formas de organización y vinculación con los servicios y los centros de la institución.

En aquellas instituciones donde la organización del PAT expresa una relación piramidal con el resto de la institución (UA-01; UA-02; UA-03; UA-04), las autoridades aparecen como responsables máximos de la acción tutorial (a las que hay que rendir cuentas anualmente) pero, en general, no se vinculan más allá de sus funciones delegando responsabilidades a quien corresponda. Por el contrario, si el PAT diseña un sistema de relaciones de colaboración e implicancia con las autoridades universitarias, éstas serán parte del desarrollo e implementación de la acción tutorial, así como de los procesos de evaluación del PAT y de formación del profesorado (UA-05; UA-06).

\section{Ideal de Buenas Prácticas}

La última categoría emergente está relacionada con las proyecciones que las unidades de análisis tienen de sus PAT para el futuro, aunque se debe advertir que atañe a la percepción de los informantes y que en ninguna de estas instituciones se encontró un plan estratégico a largo plazo de la acción tutorial, a excepción de la UA-05 que ha incorporado los elementos del PAT al Plan de Desarrollo Institucional.

En general, el ideal de buenas prácticas se puede resumir en las siguientes cuestiones, que parecen habitualmente en los discursos de los entrevistados:

- Respecto de la evaluación del PAT: mejorar los procesos de evaluación y seguimiento haciendo uso de estrategias cuantitativas y cualitativas de obtención de datos; instaurar procesos de caracterización inicial de las cohortes de ingreso (evaluación diagnóstica) para 
adaptar el PAT a tiempo; y evaluar el impacto de la acción tutorial en la evolución académica, personal y profesional de los estudiantes.

- Respecto al sistema de vinculaciones del PAT: generar mayores relaciones con los servicios y profesionales de la orientación y la psicología, de la gestión de calidad y la movilización de estudiantes; e integrar con mayor protagonismo el sistema de mentorías, con el fin de integrar otras acciones y actuaciones al PAT haciéndolo más global.

- Respecto de estrategias de mejora para el PAT: asignar a las tutorías el mismo valor (en créditos) que tienen las asignaturas; mejorar el material y la formación docente; integrar a estudiantes usuarios en la organización que sustenta el PAT y en el proceso de difusión de las tutorías; y generar orientaciones adyacentes vinculadas a la vida universitaria más allá de lo académico (salud, política, cultura, movilización, etc.).

Para finalizar este punto y a modo de resumen, se puede señalar que se han identificado las estructuras básicas de funcionamiento y organización de estas seis universidades respecto del modo cómo se gestiona y desarrolla la acción tutorial al interior de ellas. Del mismo modo, se han podido identificar aquellos temas o categorías destacados con mayor frecuencia por los informantes, sea por su magnitud en frecuencias o sea por la pertinencia y la relación con el objetivo central de este estudio, generando una red de implicancias explicitadas en la figura 1.

Si la implicancia de las autoridades es de carácter colaborativo, mayor es la cadena de compromiso institución - profesores - estudiantes que impacta directamente en la cultura universitaria y en la adopción de un sistema de evaluación claro y explícito de la acción tutorial, que genere cambios para la formación y el soporte de los tutores de la universidad.

FIGURA 1. Redes de implicancia de la acción tutorial a través del PAT

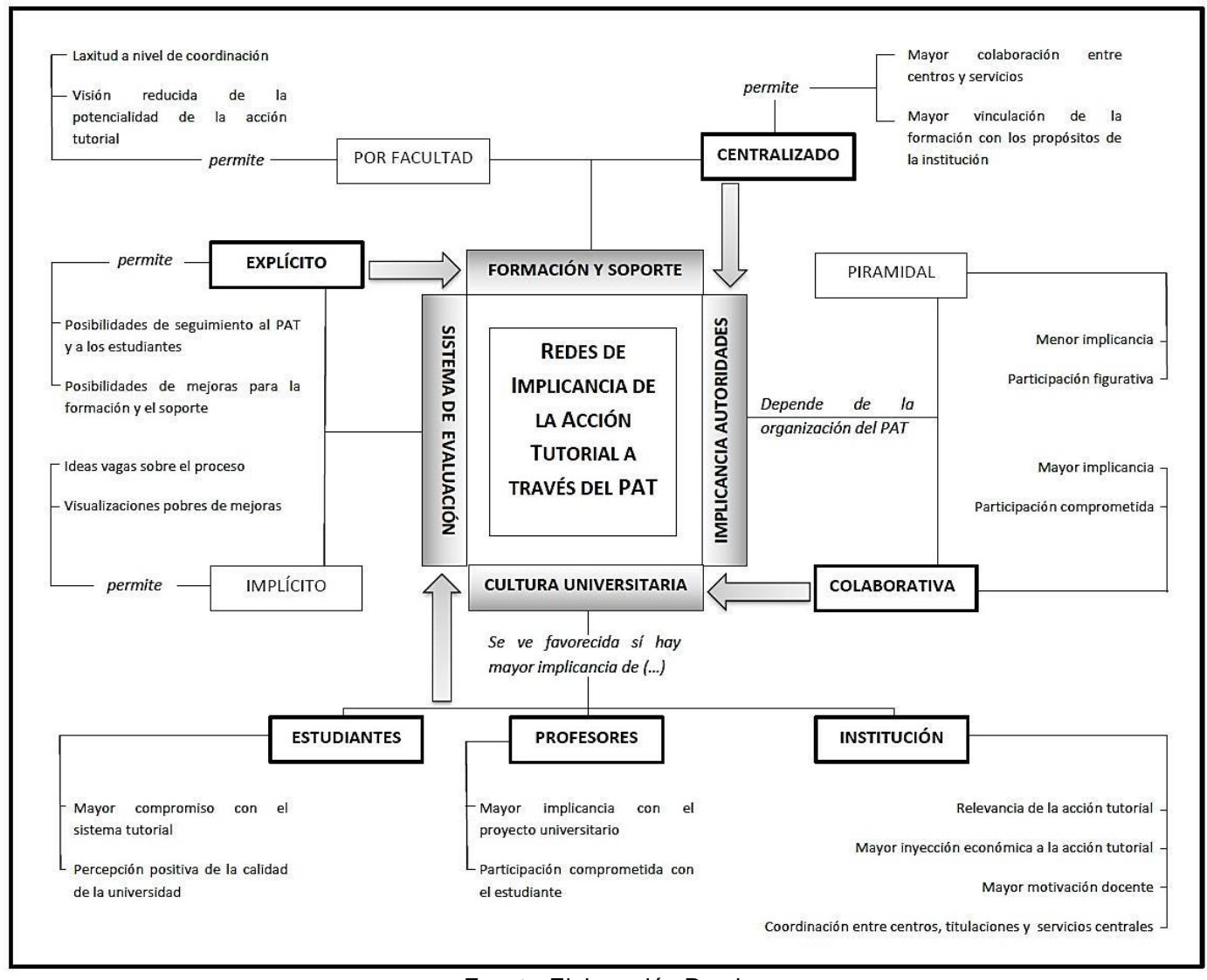

Fuente: Elaboración Propia. 


\section{Conclusiones y discusión}

El estudio analiza como las universidades participantes seleccionadas (6) desarrollan sus PAT, a partir de informantes vinculados al diseño, la conducción y desarrollo de los mismos en sus respectivas instituciones. Aunque se puede hablar de un número discreto de informantes (9), como aspecto limitante de este estudio, se ha justificado esta decisión metodológica en función de la riqueza de las aportaciones precisas de autoridades y responsables institucionales, ejecutores y formadores involucrados en el desarrollo de la acción tutorial de las unidades de análisis estudiadas

Los resultados de la investigación evidencian aquellas concepciones que poseen los informantes respecto de la naturaleza y las características básicas de la acción tutorial y las dificultades que han tenido para su desarrollo y que influyen directamente en el diseño y la puesta en marcha de los PAT al interior de las instituciones.

En este sentido, los polos que sustentarían el despliegue efectivo de los PAT serían principalmente la organización de los agentes responsables del mismo, lo que depende en gran medida del nivel de implicancia de las autoridades universitarias y de la cultura organizativa; y el compromiso de los agentes directos de la acción tutorial (docentes - estudiantes), que depende de la cultura docente y la cultura estudiantil, lo que coincide con las referencias estudiadas en Gairín et al., (2005) y Cano (2008) quienes explican que el éxito del diseño e implantación de los PAT dependen del sistema organizativo de la universidad.

Sería posible afirmar entonces, que las instituciones que presentan una organización piramidal para el PAT y una cultura docente y estudiantil poco implicada en el proceso de acción tutorial, evidencian un soporte dependiente de cada facultad o centro, un modelo de orientación más ligado al counseling y con avances hacia el modelo de programas, y un sistema de evaluación y seguimiento inexistentes 0 , a lo más, de forma indirecta a través de las asignaturas.

Por otro lado, en las universidades donde la organización para el PAT y la cultura docente y estudiantil tienen carácter colaborador e implicado, se evidencian soportes en base a comisiones inter estamentarias y/o a cargo de un servicio o unidad especializada en orientación universitaria a nivel institucional, un modelo de orientación basado en programas integrados al currículum y de servicios, que no desmerece las posibilidades del modelo clínico cuando así se estima conveniente, como también, un proceso de evaluación directo de la acción tutorial a través de sus usuarios y los tutores, mediante diversas estrategias entre las cuales destaca el uso de aplicativos intranet.

Junto a lo anterior, al establecer un análisis más abstracto y deductivo a la vez, van apareciendo elementos comunes entre las categorías de análisis iniciales que generan un constructo central de las perspectivas de significado que los informantes que han aportado a este estudio y que pueden significar referencias para la construcción y mejora de modelos de intervención sobre tutoría universitaria. Estos aspectos son:

- La necesidad de (re) validación de la acción tutorial y del PAT: Algunos aspectos (falta de evaluación, nivel de implicancia de los agentes involucrados, formas puramente administrativa de organizar el plan, inyección económica, remuneraciones etc.) que indican la necesidad de volver a revisar el papel que desempeña la acción tutorial en la universidad a través del PAT, tal y como proponen, Alcolea y Pérez (2012), Álvarez y Álvarez (2015) y Álvarez (2014; 2017). 
Estos autores señalan que existen déficits en el modelo tutorial actual (tal como se planteaba en las referencias teóricas de esta investigación) que deben ser mejorados como: que el sistema organizativo se basa en el modelo clásico de dirección administrativa (lo que no sería suficiente) y que se requieren cambios efectivos en el diseño y concreción de los PAT, puesto que éste responde adecuadamente a un contexto de formación por competencias y a la validez ecológica, personal, formativa y normativa que caracteriza al proceso educativo. Además se ha de tener en cuenta que la tutoría universitaria se considera hoy en día como un elemento de calidad (Clares, Juárez y Cusó, 2016) por lo que conviene replantearse esta cuestión.

- La necesidad de (re) incorporar la acción tutorial y el PAT a la Cultura Universitaria: Hacemos referencia a los dilemas actuales vinculados a la implementación de la acción tutorial al interior de la universidad respecto de la cultura interna de las propias instituciones, donde el desarrollo del PAT debería impactar en las creencias, los valores, las presunciones básicas y los comportamientos de la organización, los docentes y los estudiantes de manera positiva, para ser considerado efectivamente como un elemento más de la formación, perfectible e integrada en el currículum, y no un servicio aislado y estático que depende sólo del tutor. En palabras de Cano (2008, p.191), cuatro deben ser las condiciones que, a priori, se han de dar para que el cambio sea posible: i) Creación y regulación de un marco institucional de carácter organizativo- aplicativo con capacidad de pervivencia; ii) Cambio pedagógico-didáctico en las actitudes, mentalidad y modos de hacer de profesores y alumnos; iii) Cambio pedagógico en la cultura universitaria de todos sus miembros y, fundamentalmente, de sus órganos unipersonales y colegiados de decisión; y iv) Inclusión de las medidas adoptadas, como un criterio de evaluación de la calidad de las universidades, en los procesos de evaluación y acreditación llevados a cabo tanto por las agencias nacionales como por las autonómicas.

- La necesidad de mejorar las prácticas de gestión de los PAT: las percepciones recogidas relevan la importancia de gestionar los planes de acción tutorial mejor de como se viene haciendo hasta el momento, reconociendo sus aciertos y debilidades, y cómo éstas están vinculadas a aspectos de la cultura interna de la institución y a la reformulación de sus acciones. Se destaca que el PAT debe ir más allá de su reconocimiento institucional como "instrumento de apoyo a la formación universitaria" y que debiera pasar a ser una función institucional que se incluya tanto en los programas formativos de las titulaciones como en el Plan de Desarrollo Estratégico de cada institución.

Álvarez (2014) destaca, en este sentido, que se ha de generar un espacio de convergencia tutorial en las instituciones de enseñanza superior que debe iniciarse con un reconocimiento institucional de la orientación y acción tutorial como un componente fundamental de la función docente. Así, debe constar en el plan de dedicación docente como una acción prioritaria que contribuya al desarrollo integral del alumnado, lo que implica la adecuación del peso curricular -ECTS- a las demandas de trabajo, la posibilidad de desarrollo y evaluación de las competencias, la coordinación de la disciplina y la creación de espacios interdisciplinares específicos. Acto seguido, "se han de crear espacios de convergencia tutorial entre: i) los servicios de orientación universitaria; ii) la coordinación académica; iii) la coordinación de tutoría; iv) la tutoría de asignatura; v) la tutoría de la carrera o de acompañamiento académico-profesional; vi) la tutoría de practicum; vii) y la tutoría de iguales" (p.17).

La información obtenida evidencia cómo el sistema universitario de estos seis centros, caracterizado por sus culturas internas, beneficia o interfiere en el desarrollo de la acción tutorial, generándose así, una red de implicancias basada en el compromiso de las autoridades, los 
profesores y estudiantes, que incide en la cultura universitaria, en el sistema de evaluación y de formación y soporte de la acción tutorial.

No obstante, su actividad condiciona la necesidad de reformular los Planes de Acción Tutorial y de generar un modelo de gestión que dé soporte al PAT y que responda a las necesidades de los estudiantes y al contexto de las titulaciones, las facultades y los centros formativos en general.

Finalmente, el estudio nos permite plantear interrogantes que abren nuevas líneas de investigación relacionadas con la gestión de la acción tutorial en otras latitudes como lo son los países latinoamericanos, que se han servido de la experiencia española para generar programas tutoriales de cara a la atención integral y la permanencia de los estudiantes en la enseñanza superior.

\section{Referencias bibliográficas}

Alcolea, G. \& Pérez, M. J. (2012). Un paso más allá en la planificación docente uni-versitaria: el plan de acción tutorial. Estudios sobre el Mensaje Periodístico, 18 (Núm. especial octubre 2012), 45-55. http://doi.org/10.5209/rev_ESMP.2012.v18.40889

Álvarez, C. \& San Fabián, J. L. (2012). La elección del estudio de caso en investigación educativa. Gazeta de Antropología, 28(1), 1-13. Retrieved from http://hdl.handle.net/10481/20644

Álvarez González, M. (2017). Hacia un modelo integrador de la tutoría en los diferentes niveles educativos. Educatio Siglo XXI, 35(2), 21-42. http://doi.org/10.6018/j/298501

Álvarez González, M. \& Álvarez Justel, J. (2015). La tutoría universitaria: del modelo actual a un modelo integral. Revista Electrónica Interuniversitaria de Formación Del Profesorado, 18(2), 125-142. http://doi.org/10.6018/reifop.18.2.219671

Álvarez, M. (2014). La orientación y la tutoría en la formación de grados y postgrados. In P. Figuera (Ed.), Persistir con éxito en la universidad: de la investigación a la acción. (pp. 175 203). Barcelona: Laertes.

Blanchard, M. (2010). ¿Tiene sentido la Acción Tutorial en la Universidad? Una estrategia educativa a favor del estudiante y para la mejora de la calidad. Revista Crítica, (969), 70 - 75.

Blasco Calvo, P. \& Pérez Boullosa, A. (2012). Enfoques y aplicaciones prácticas en Orientación y Acción Tutorial. Valencia: Nau Llibres. Recuperado de https://books.google.es/books?hl=es\&lr=\&id=ew_octTVxZ8C\&oi=fnd\&pg=PA9\&dq=Enfoques+ $\mathrm{y}+$ aplicaciones+pr\%C3\%A1cticas+en+Orientaci\%C3\%B3n+y+Acci\%C3\%B3n+Tutorial\&ots=r HqTxrLuFp\&sig=AqAIQmC5E-hj4P3k86mRbQWDAAo

Cano, R. (2008). Modelo organizativo para la planificación y desarrollo de la tutoría universitaria en el marco del proceso de convergencia europea en Educación Superior. Revista Interuniversitaria de Formación Del Profesorado, ISSN 0213-8646, No 61, 2008 (Ejemplar Dedicado a: La Tutoría Universitaria en el Espacio Europeo de Educación Superior / Coord. Por Rufino Cano González), Págs. 185-206, (61), 185-206.

Clares, P. M., Juárez, M. M. \& Cusó, J. P. (2016). ¿Cómo avanzar en la tutoría universitaria? Estrategias de acción: los estudiantes tienen la palabra. REOP - Revista Española de Orientación y Psicopedagogía, 27(2), 80-98.

Creswell, J. W. (2013). Qualitative inquiry research design choosing among five approaches (3rd ed.). Thousand Oaks, CA: Sage Publications, Ltd. 
Da Re, L. (2013). Orientación durante el periodo formativo en la universidad: La tutoría en Italia y Padova. En XVI Congreso Nacional / II Internacional Modelos de Investigación Educativa de AIDIPE. Alicante: AIDIPE. Recuperado de https://www.academia.edu/8513350/La_orientaci\%C3\%B3n_en_la_educaci\%C3\%B3n_superi or_una_visi\%C3\%B3n_internacional

De La Rubia, J., Tur, M., Paz, M., Álvarez Azaustre, M. \& Guillem, P. (2013). Tutorías como respuesta en la Orientación Universitaria. In $X$ Jornadas Internacionales de Innovación Universitaria, Educar para Transformar. Retrieved from http://abacus.universidadeuropea.es/bitstream/handle/11268/2683/0006.pdf?sequence=1

Duarte, S. R., Vasconcelos, P. V., Asencio, E. N., Martínez, A. M. M. \& Laguna, A. P. (2012). Orientación educativa: fundamentos teóricos, modelos institucionales y nuevas perspectivas. Recuperado de https://books.google.com/books?id=8CwbAgAAQBAJ\&pgis=1

Figuera, P. \& Álvarez, M. (2014). la intervención orientadora y tutorial en la adaptación y persistencia del alumnado en la universidad. Revista de Orientación Educacional, 28(54), 3149. Recuperado de http://www.roe.cl/index.php/roe/article/view/83/40

Folch, M. T. \& Ion, G. (2008). Contrastando dos modelos de análisis de la cultura organizativa de la universidad. Bordón, 60(3), 131 - 147.

Gairín, J., Feixas, M., Franch, J., Guillamón, C. \& Quinquer, D. (2003). Elementos para la elaboración de planes de tutoría en la universidad. Contextos Educativos, (6), 21 - 42.

Gairín, J., Guillamón, Rc., Quinquer, D. \& Feixas, M. (2005). El plan de tutoria a la Universitat. Barcelona: Servei de Publicacions Universitat Autònoma de Barcelona.

Hernández, R., Fernández, C. \& Baptista, P. (2010). Metodología de la Investigación (Quinta edición). México D.F.: Mc Graw - Hill Interamericana.

Hernández, R., Fernández, C. \& Baptista, P. (2006). Metodología de la Investigación. Metodología de la investigación (Cuarta Edición). Iztapalapa, México D.F.: McGraw - Hill Interamericana.

Luck, C. (2010). Challenges faced by tutors in Higher Educatione. Psychodynamic Practice, 16(3), 273-287. http://doi.org/10.1080/14753634.2010.489386

Martínez Clares, P., Martínez Juárez, M. \& Pérez Cusó, J. (2014). Tutoría universitaria: Entorno emergente en la Universidad Europea. Un estudio en la Facultad de Educación de la Universidad de Murcia. Revista de Investigacion Educativa, 32(1), 111-138. http://doi.org/10.6018/rie.32.1.148411

Rodríguez, S. (2004). Manual de Tutoría Universitaria. Recursos para la acción. Barcelona: Octaedro: ICE - UB.

Rumbo, B. \& Gómez, T. (2011). La acción tutorial en un contexto universitario masificado y la reivindicación europea de su valor formativo. Revista de Formación e Innovación Educativa Universitaria, 4(1), 13-34.

Sanz, R. (2005). Integración del estudiante en el sistema universitario, La tutoría. Cuadernos de Integración Europea, (2), 69-95. Recuperado de http://www.cuadernosie.info

Sanz, R. (2009). Tutoría y Orientación en la Universidad. En Tutoría y atención personal al estudiante en la universidad (pp. 45-70).

Traverso, A. (2015). Le competenze educative del tutor dell'orientamento in ambito scolastico e universitario. Pedagogia Oggi, (1), 254-267. Recuperado de https://www.researchgate.net/publication/281637498_Le_competenze_educative_del_tutor_d ell'orientamento_in_ambito_scolastico_e_universitario_The_Educational_competencies_of_th e_Guidance_Tutor_in_the_Area_of_Schoool_and_University 
Vieira, M. J. (2007). Influencia comparada del perfil de los estudiantes en la planificación de la orientación universitaria. Revista Española de Pedagogía, (236), 49-70. Recuperado de http://reunir.unir.net/bitstream/handle/123456789/4034/influenciaComparadaDelPerfilDeLosEs tudiantes.pdf?sequence $=1 \&$ isAllowed $=y$

\section{Fuentes electrónicas}

Riquelme, M. (2014). Las 5 Funciones de la Administracion de Henri Fayol. Recuperado el 4 de mayo de 2015, dehttps://www.webyempresas.com/las-5-funciones-de-la-administracion-dehenri-fayol/

Fecha de entrada: 29 Marzo 2017

Fecha de revisión: 17 Marzo 2018

Fecha de aceptación: 20 Marzo 2018 